\title{
Cryoballoon Catheter Ablation in Atrial Fibrillation
}

\author{
Cevher Ozcan, Jeremy Ruskin, and Moussa Mansour \\ Cardiac Arrhythmia Service, Heart Center, Massachusetts General Hospital, Boston, MA 02114, USA \\ Correspondence should be addressed to Moussa Mansour, mmansour@partners.org
}

Received 16 December 2010; Revised 5 March 2011; Accepted 25 April 2011

Academic Editor: Atul Verma

Copyright () 2011 Cevher Ozcan et al. This is an open access article distributed under the Creative Commons Attribution License, which permits unrestricted use, distribution, and reproduction in any medium, provided the original work is properly cited.

\begin{abstract}
Pulmonary vein isolation with catheter ablation is an effective treatment in patients with symptomatic atrial fibrillation refractory or intolerant to antiarrhythmic medications. The cryoballoon catheter was recently approved for this procedure. In this paper, the basics of cryothermal energy ablation are reviewed including its ability of creating homogenous lesion formation, minimal destruction to surrounding vasculature, preserved tissue integrity, and lower risk of thrombus formation. Also summarized here are the publications describing the clinical experience with the cryoballoon catheter ablation in both paroxysmal and persistent atrial fibrillation, its safety and efficacy, and discussions on the technical aspect of the cryoballoon ablation procedure.
\end{abstract}

\section{Introduction}

Atrial fibrillation (AF) is the most common sustained cardiac rhythm disturbance, increasing in prevalence with age [1]. Pulmonary vein (PV) isolation with catheter ablation is one of the primary treatments for symptomatic AF refractory or intolerant to antiarrhythmic medications [1]. In some cases, it may be even appropriate to perform AF ablation as first-line therapy, particularly in selected symptomatic patients with heart failure and/or reduced left ventricular ejection fraction [2]. The main goal in catheter ablation is complete electrical isolation of the PVs, and therefore the success of the procedure and the reduction of procedurerelated complications require careful identification of the PV ostia and application of ablation to the PV antra.

Currently radiofrequency (RF) energy is the most commonly used energy source for ablation. Many studies demonstrated the efficacy of RF energy for the treatment of AF. However, this form of energy is associated with various complications including thromboembolic events, PV stenosis, and atrio-esophageal fistula [3]. Thus, new energy sources have been investigated for the treatment of AF. These include cryothermal energy, ultrasound, and laser [4]. Recent clinical and preclinical studies demonstrated that the cryothermal energy application using a balloon catheter for $\mathrm{PV}$ isolation is an effective treatment for $\operatorname{AF}[5,6]$.

The purpose of this paper is to review the current literature for better understanding of the utility of cryoablation for the treatment of patients with AF. The literature was searched in PubMed from 1950 to February 2011 with the keywords "atrial fibrillation," "cryosurgery," and "catheter ablation" published in English. A total of 100 references were found. All full-text articles and their references were reviewed and included in this paper if they were relevant to our presentation.

\section{Effects of Cryothermal Energy at the Cellular Level}

Cryothermal energy causes progressive necrosis in the absence of significant alteration of tissue structure at thaw. The effect of ablation on the cellular ultrastructure has been evaluated in studies using a cryoprobe cooled to $-60^{\circ} \mathrm{C}$ with expanding nitrous oxide [7]. Ice crystals were observed to form inside the cells during ablation, with a resulting cell damage that is osmotic rather than mechanical [7]. Microcirculatory changes have also been described leading to tissue necrosis. Acute changes in subcellular organelle structure and mitochondrial destruction occur in 1 minute at $-70^{\circ} \mathrm{C}$. The mitochondria appear enlarged, with decreased matrix density and disrupted cristae. Striking depletion of glycogen stores takes place within 1 hour of thaw. Subsequent changes up to 10 hours after thaw are most notable for progressive mitochondrial damage. Then, the hemorrhagic and inflammatory phase starts and results in fibrosis. The cryothermal 
energy lesion formation can be divided in three sequential stages which are the freeze/thaw phase, the hemorrhagicinflammatory phase, and the replacement fibrosis phase. The resulting lesions are minimally thrombogenic, have sharp well-demarcated margins, and exhibit minimal tissue disruption and preserved basic underlying tissue architecture $[8,9]$. Thus the histopathologic changes of cryoablation are likely to be associated with low arrhythmogenic potential.

\section{Clinical Application of Cryoablation}

Cryothermal energy has been used in treatment of cardiac arrhythmias for the past 3 decades, especially in supraventricular arrhythmias. Cryoablation was performed as early as 1977 to the atrioventricular (AV) conduction system [10]. Harrison et al. studied cryoablation using a handheld nitrous oxide-cooled cryogenic probe in 20 dogs and subsequently in three patients with drug-resistant, lifethreatening supraventricular tachycardias [10]. In the same year accessory pathway elimination by cryoablation was demonstrated by Gallagher et al. [11]. Since then, numerous experimental and clinical studies have evaluated the efficacy and safety of cryoablation [4, 5, 9, 12-14].

A multicenter clinical trial in patients with AV nodal reentry tachycardia (103 patients), AV reentrant tachycardia (51 patients), and AF (12 patients) demonstrated that cryoablation is a safe and effective strategy for the treatment of supraventricular arrhythmias [12]. Catheterbased cryoablation was found to be a safe alternative to RF ablation for slow pathway modification, accessory pathway termination, and AV junction ablation. Acute procedural success was achieved in $83 \%$ of the overall group, and long-term success after 6 months was 91\%. Cryomapping (cooling the catheter tip to $-30^{\circ} \mathrm{C}$ ) successfully identified ablation targets in the majority of the patients, and the electrophysiologic effects of cryomapping were reversible within minutes in $94 \%$ of the attempts.

Also, a prospective, randomized trial in patients with recurrent narrow QRS-complex tachycardia suggestive of AV nodal reentry tachycardia compared cryoablation with RF ablation and showed a procedural success rate of $91 \%$ in the RF group and $93 \%$ in the cryoablation group [13]. The median number of cryothermal applications was significantly lower than the number of RF applications. Both fluoroscopy and procedural times were comparable. No cryoenergy-related complications were observed, and no permanent AV conduction block occurred. Long-term clinical success was comparable in both groups.

Currently cryoablation is used to treat various forms of ventricular and supraventricular arrhythmias including AV nodal reentry tachycardia, AV reentrant tachycardia, atrial flutter, and focal atrial tachycardia [12-18]. Compared to RF ablation, cryoablation has been associated with lack of pain during energy delivery, homogenous lesion formation, less destruction to surrounding vasculature, preserved tissue integrity, and lower risk of thrombus formation [5-9, 1214]. Lesion reversibility is another advantage of cryoablation over RF energy and is useful for ablation of accessory pathways in proximity to the conduction system [1215]. Moreover, cryoablation can be applied within venous structures, like the coronary sinus and the middle cardiac vein with less risk of thrombosis compared to RF ablation $[14,19]$. It has been shown that cryoablation in the coronary sinus within $2 \mathrm{~mm}$ of the left circumflex artery produces myocardial lesions similar to RF ablation with lower risk of coronary artery stenosis [19].

The absence of endothelial disruption with cryoablation results in less thrombogenicity and may offer an opportunity for improved safety during ablations in the left side of the heart [5-9]. This may be important when large areas of the endocardium are ablated such as in AF ablation procedures. All these characteristics of cryoenergy, in addition to the ability to use it with a balloon, make it an attractive source of energy for PV isolation.

\section{Balloon-Based Ablation Catheter}

Point-to-point ablation using a focal catheter for PV isolation is associated with technical difficulties and requires experienced operators with high level of skills. Pulmonary vein isolation using a focal cryocatheter has been studied and was found to be associated with long procedure times [20]. The cryoballoon catheter was designed with the aim of facilitating PV isolation [4-6, 21].

\section{Cryoballoon for Pulmonary Vein Isolation}

5.1. Ablation Technique. Currently only one balloon design is available for clinical use (Medtronic Inc, Minnesota, Minn). This system includes an over-the-wire balloon catheter which is cooled using nitrous oxide $\left(\mathrm{N}_{2} \mathrm{O}\right)$ [4-6, 22-27]. The balloon shaft size is $10.5 \mathrm{~F}$ and has a deflection mechanism. It is introduced into the left atrium using a 14 Fr steerable sheath. The shaft has a central lumen that can accommodate a wire for support and also used for contrast and saline injection.

The wire is used to engage the vein first. The balloon is inflated outside the vein and advanced over the wire to occlude the vein (Figure 1). Obtaining a complete occlusion is crucial for successful isolation. An incomplete occlusion will lead to flow around the balloon and prevents the temperature from reaching low enough levels necessary for adequate ablation. A complete occlusion is typically confirmed by injecting contrast in the lumen at the balloon tip. Once occlusion is confirmed, the contrast is flushed using saline and freezing is started. If successful, a dip in the temperature curve is observed indicating a better occlusion. Lesions are usually $240-300 \mathrm{sec}$ long and PV isolation is usually achieved with one lesion in most patients [22-24]. Most operators apply one additional lesion after isolation.

Currently, two sizes of the cryoballoon catheter are available, $28 \mathrm{~mm}$ and $23 \mathrm{~mm}$ diameter. It is advisable to use a $28 \mathrm{~mm}$ balloon regardless of the size of the vein in order to reduce the risk of complications such as PV stenosis and phrenic nerve injury. A quadripolar catheter is usually positioned in the superior vena cava for continuous 


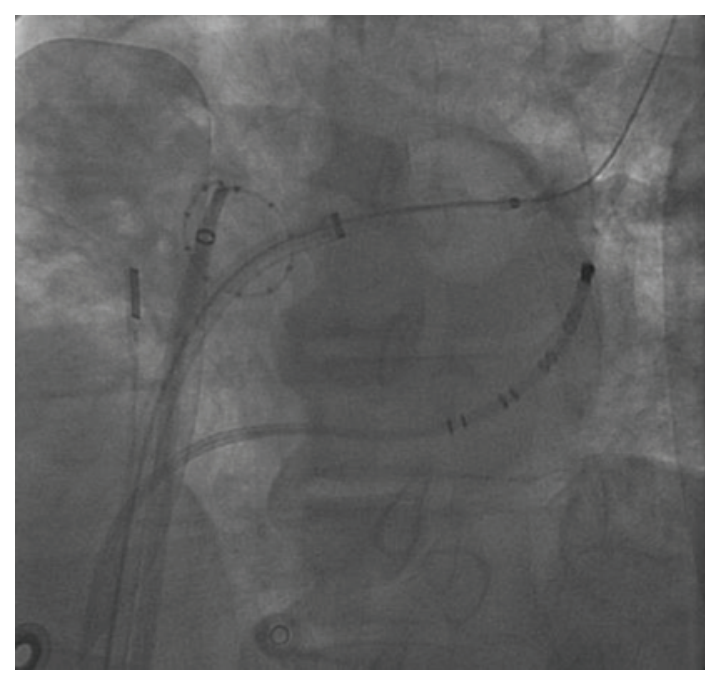

FIGURE 1: Cryoballoon catheter placement for pulmonary vein isolation with fluoroscopic A. Fluoroscopic appearance $\left(30^{\circ}\right.$ left anterior oblique view) of the cryoballoon inflated in a left superior pulmonary vein. The guidewire was advanced in a pulmonary vein branch, and the occlusion is confirmed by the absence of flow during contrast injection in the vein. The circular mapping catheter is placed at the ostium of the right superior pulmonary vein. A multipolar catheter in the coronary sinus and an intracardiac echocardiography catheter in the mid-right atrium are visible.

phrenic nerve stimulation during cryoablation of right PVs. Intracardiac echocardiogram and color Doppler can be used to confirm occlusion and the site of gaps if present (Figure 2). Recently, the use of transesophageal echocardiography with color Doppler was described allowing real-time visualization of the cryoballoon during ablation [28].

After ablation, PV isolation is assessed using a circular mapping catheter inserted in the left atrium via a separate transseptal puncture $[22,25,29]$. A small caliber circular mapping that can be advanced in the central lumen instead of the wire can also be used to confirm isolation.

In some patients technical challenges can be encountered leading to the inability to obtain a complete occlusion. Two maneuvers can be helpful if complete occlusion cannot be achieved by advancing the balloon. The first involves trying to engage a different branch with the wire. This can provide a better alignment of the catheter shaft with the axis of the PV. The second maneuver is the "pull-down" technique [24]. This can be useful when a gap is present at the inferior border of the vein and cannot be closed despite advancing the balloon. With this technique, freezing is started despite the presence of the gap. At about 60-90 sec into the freeze, the balloon and the sheath are both pulled down gently in order to close the gap at the inferior portion of the vein. This should be done very carefully because of the risk of severe vascular damage if excessive force is applied. If occlusion and isolation cannot be obtained despite these maneuvers, a standard point-by-point ablation catheter can be used for completion.

The sites of PV reconnection have been analyzed in some studies [24, 29-31]. The inferior border of the veins as well

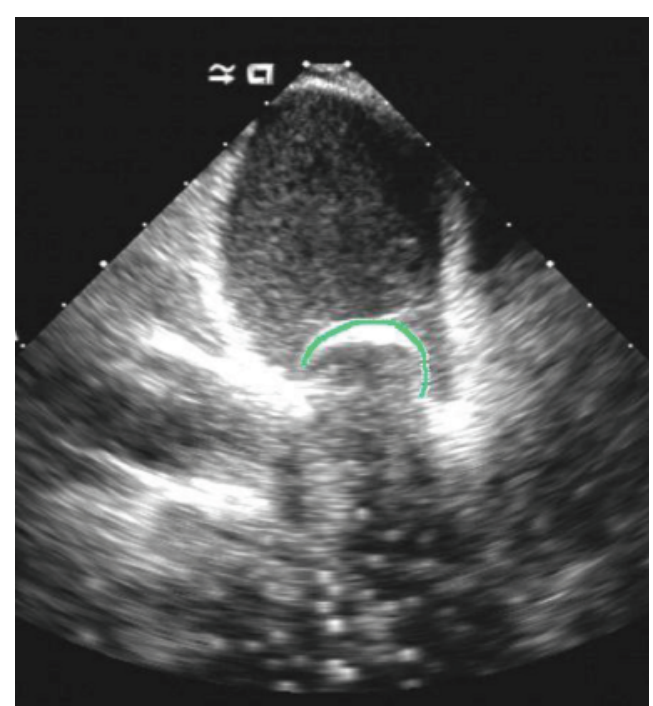

Figure 2: An intracardiac echocardiographic image demonstrates total occlusion of left superior pulmonary vein by cryoballon catheter.

as the ridge between the left atrial appendage and the left PV was found to be the sites where most reconnections occurred. This is likely to be the result of sharp catheter angulation with loss of central cryoballoon alignment with the axis of the PVs.

5.2. Clinical Results: Efficacy of the Procedure. To date over 10,000 PV procedures have been performed worldwide. There have been many publications describing the clinical experience with the cryoballoon in both paroxysmal and persistent AF [22-41].

An early report by Van Belle and colleagues described 57 consecutive patients with paroxysmal AF who underwent cryoballoon ablation [22]. Eighty-four percent of the targeted PVs were successfully isolated using the cryoballoon and the rest required a standard cryocatheter to achieve isolation. A daily monitoring of rhythm demonstrated a significant reduction in AF burden. Klein et al. reported the result of cryoballoon ablation in 21 patients with symptomatic paroxysmal AF in 2008 [26]. A total of $95 \%$ of PVs were isolated, and $86 \%$ of the patients remained free of AF after 6 months based on periodic holter monitoring. Subsequently, Neumann et al. evaluated the efficacy of cryoballoon ablation in 293 patients with paroxysmal and 53 patients with persistent AF [23]. Ninety-seven percent of the PVs were isolated with either cryoballoon alone or in combination with a standard cryocatheter. Maintenance of sinus rhythm was reported in $74 \%$ and $42 \%$ of the patients with paroxysmal and persistent AF, respectively. In a recent study by Ahmed et al. [24], the permanence of PV isolation after cryoballoon ablation was tested in 12 patients with paroxysmal AF. Eighty-eight percent of PVs remained isolated at 8-12 weeks after the initial procedure.

The most comprehensive result regarding the efficacy and safety of the cryoballoon catheter ablation for the 
treatment of AF was obtained from the STOP-AF trial $[32,33]$. This trial randomized patients with paroxysmal drug-refractory AF in a 2:1 fashion to either PV isolation with cryoballoon ablation or antiarrhythmic drug therapy. Balloon-only isolation of PVs was achieved in $90.8 \%$, and the overall procedural success ( $\geq 3 \mathrm{PVs}$ isolated) was achieved in $98.2 \%$ of the patients. Nineteen percent of the patients needed a repeat cryoablation procedure within the 90-day blanking period. The success rate at 12-month followup was $69.9 \%$.

The use of cryoballoon for PV isolation in patients with persistent AF has been associated with high rates of arrhythmia recurrence $[23,34,35]$. This is not surprising knowing that the left atrial substrate and not only the PVs play a role in AF maintenance in this subgroup of patients. A study from our group investigated the combined use of cryoballoon PV isolation and conventional RF ablation of areas of complex fractionated electrograms in 22 patients with persistent AF [27]. After a single procedure, $86 \%$ of patients were AF-free at 6-month followup.

In this study the followup was performed in most patients using implantable loop monitors allowing automatic detection of asymptomatic AF. In addition to AFfree survival, AF burden reduction was also analyzed in some studies. Van Belle et al. studied 144 patients with symptomatic AF refractory to antiarrhythmic drugs who underwent cryoballoon PV isolation [36]. Followup was performed using daily transtelephonic ECG monitoring, $24 \mathrm{~h}$ Holter-ECG, and an arrhythmia-focused questionnaire to document AF. After ablation, AF burden was reduced from $26 \%$ to $9 \%$.

5.3. Complications of Cryoballoon Catheter Ablation. Phrenic nerve palsies (PNP), PV stenosis, and other complications have been reported with cryoballoon PV isolation [22-41]. In the STOP AF study, PNP was reported in 29 out of 259 procedures $(11.2 \%)[32,33]$. Of these, only four $(13.8 \%)$ patients had persistent PNP at 12 months. PNP can occur with isolation of the right superior and less commonly the right inferior PV.

Another important procedural complication with the cryoballoon is PV stenosis. Although earlier studies showed no significant incidence of PV stenosis following cryoballoon ablation, STOP-AF trial demonstrated 3.1\% risk of PV stenosis in its study population $[32,33]$. This complication is likely associated with ablation of the tubular portion of the vein. As a result, it is believed that the use of the $28 \mathrm{~mm}$ balloon can reduce this complication because this larger balloon is less likely to be positioned inside the vein during ablation [25]. For the same reason, it is believed that the use of the larger balloon will carry a lower risk of PNP.

There are no reports of atrioesophageal fistula associated with the use of the cryoballoon. The effect of cryoballoon PV isolation on the esophagus has been studied [37]. Luminal esophageal temperature was significantly decreased (>1 degrees C) in 62 of 67 (93\%) patients, and reversible esophageal injuries were seen in $17 \%$ of patients. However, no atrial-esophageal fistula was documented and the followup endoscopy confirmed healing of all ulcers.
Other procedure-related complications with the use of the cryoballoon are similar to RF ablations including stroke, pericardial effusion or tamponade, femoral vein access complications, and cerebral embolism [22-41].

5.4. Comparison with Conventional Radiofrequency Catheter Ablation. Both conventional focal RF ablation and cryoablation are used for $\mathrm{PV}$ isolation and both are shown to be effective [1-3, 22-44]. Recent studies compared clinical outcome of cryoballoon ablation for PV isolation with RF ablation in regards to the safety and the efficacy of the procedure [30, 35, 42].

Linhart et al. demonstrated in a case-control study of 40 patients with paroxysmal atrial fibrillation that cryoballoon ablation has similar success rate to RF ablation in addition to similar procedure and fluoroscopy times [42]. Kojodjojo et al. studied the efficacy of a strategy using a large cryoballoon to perform antral PV isolation in 124 patients with paroxysmal and persistent AF [35] and compared it with RF ablation. At one-year followup, 77\% of paroxysmal and $48 \%$ of persistent AF patients remained free from AF after a single procedure. In the RF group $72 \%$ of the patients with paroxysmal remained free of AF. The procedural and fluoroscopic times with cryoablation were shorter than RF ablation. A recent study from Kühne at al. confirmed the shorter procedure duration with cryoablation compared to $\mathrm{RF}$ (166 \pm 32 versus $197 \pm 52$ minutes) [30]. In this study, a total of 55 patients with paroxysmal AF were studied: 25 patients underwent PVI using a 28-mm cryoballoon and 25 patients using an open-irrigation RF catheter. The number of procedures was not different in the 2 groups $(1.2 \pm 0.4$ in cryoablation versus $1.3 \pm 0.6$ in RF ablation) with a success rate at one-year followup of $88 \%$ in the cryoballoon group and $92 \%$ in the RF group. Another study, the Freeze AF trial, is being planned to compare cryoballoon catheter ablation with open-irrigation RF ablation in a randomized clinical study including 244 patients with paroxysmal AF [43].

\section{Conclusions}

Cryoballoon catheter ablation is a safe and effective technique for PV isolation to treat paroxysmal AF. Overall success rate in persistent $\mathrm{AF}$ is lower. Potential procedural complications related to cryoballoon ablation include PNP and PV stenosis as well as rare neurological complications.

\section{Acknowledgment}

This work was partially supported by the Deane Institute for Integrative Research in Atrial Fibrillation and Stroke.

\section{References}

[1] V. Fuster, L. E. Rydén, D. S. Cannom et al., "ACC/AHA/ESC 2006 guidelines for the management of patients with atrial fibrillation: a report of the American College of Cardiology/American Heart Association Task Force on practice guidelines and the European Society of Cardiology Committee for practice guidelines (Writing committee to revise the 
2001 guidelines for the management of patients with atrial fibrillation): developed in collaboration with the European Heart Rhythm Association and the Heart Rhythm Society," Circulation, vol. 114, no. 7, pp. e257-e354, 2006.

[2] H. Calkins, J. Brugada, D. L. Packer et al., "HRS/EHRA/ECAS expert consensus statement on catheter and surgical ablation of atrial fibrillation: recommendations for personnel, policy, procedures and follow-up. A report of the Heart Rhythm Society (HRS) Task Force on Catheter and Surgical Ablation of Atrial Fibrillation. Developed in partnership with the European Heart Rhythm Association (EHRA)," Heart Rhythm, vol. 4, no. 6, pp. 816-861, 2007.

[3] R. Cappato, H. Calkins, S. A. Chen et al., "Worldwide survey on the methods, efficacy, and safety of catheter ablation for human atrial fibrillation," Circulation, vol. 111, no. 9, pp. 1100-1105, 2005.

[4] V. Y. Reddy, P. Neuzil, A. d'Avila et al., "Balloon catheter ablation to treat paroxysmal atrial fibrillation: what is the level of pulmonary venous isolation?" Heart Rhythm, vol. 5, no. 3, pp. 353-360, 2008.

[5] A. V. Sarabanda, T. J. Bunch, S. B. Johnson et al., "Efficacy and safety of circumferential pulmonary vein isolation using a novel cryothermal balloon ablation system," Journal of the American College of Cardiology, vol. 46, no. 10, pp. 1902-1912, 2005.

[6] B. Avitall, D. Urboniene, G. Rozmus, D. Lafontaine, R. Helms, and A. Urbonas, "New cryotechnology for electrical isolation of the pulmonary veins," Journal of Cardiovascular Electrophysiology, vol. 14, no. 3, pp. 281-286, 2003.

[7] D. K. Whittaker, "Mechanisms of tissue destruction following cryosurgery," Annals of the Royal College of Surgeons of England, vol. 66, no. 5, pp. 313-318, 1984.

[8] D. L. Lustgarten, D. Keane, and J. Ruskin, "Cryothermal ablation: mechanism of tissue injury and current experience in the treatment of tachyarrhythmias," Progress in Cardiovascular Diseases, vol. 41, no. 6, pp. 481-498, 1999.

[9] P. Khairy, P. Chauvet, J. Lehmann et al., "Lower incidence of thrombus formation with cryoenergy versus radiofrequency catheter ablation," Circulation, vol. 107, no. 15, pp. 2045-2050, 2003.

[10] L. Harrison, J. J. Gallagher, and J. Kasell, "Cryosurgical ablation of the A $\mathrm{V}$ node his bundle. A new method for producing A V block," Circulation, vol. 55, no. 3, pp. 463-470, 1977.

[11] J. J. Gallagher, W. C. Sealy, and R. W. Anderson, "Cryosurgical ablation of accessory atrioventricular connections. A method for correction of the preexcitation syndrome," Circulation, vol. 55, no. 3, pp. 471-479, 1977.

[12] P. L. Friedman, M. Dubuc, M. S. Green et al., "Catheter cryoablation of supraventricular tachycardia: results of the multicenter prospective "frosty" trial," Heart Rhythm, vol. 1, no. 2, pp. 129-138, 2004.

[13] G. P. Kimman, D. A. M. J. Theuns, T. Szili-Torok, M. F. Scholten, J. C. Res, and L. J. Jordaens, "CRAVT: a prospective, randomized study comparing transvenous cryothermal and radiofrequency ablation in atrioventricular nodal re-entrant tachycardia," European Heart Journal, vol. 25, no. 24, pp. 22322237, 2004.

[14] K. Lemola, M. Dubuc, and P. Khairy, "Transcatheter cryoablation part II: clinical utility," PACE-Pacing and Clinical Electrophysiology, vol. 31, no. 2, pp. 235-244, 2008.

[15] F. Gaita, A. Montefusco, R. Riccardi et al., "Acute and longterm outcome of transvenous cryothermal catheter ablation of supraventricular arrhythmias involving the perinodal region,"
Journal of Cardiovascular Medicine, vol. 7, no. 11, pp. 785-792, 2006.

[16] R. Manusama, C. Timmermans, F. Limon, S. Philippens, H. J. G. M. Crijns, and L. M. Rodriguez, "Catheter-based cryoablation permanently cures patients with common atrial flutter," Circulation, vol. 109, no. 13, pp. 1636-1639, 2004.

[17] O. A. Obel, A. D’Avila, P. Neuzil, E. B. Saad, J. N. Ruskin, and V. Y. Reddy, "Ablation of left ventricular epicardial outflow tract tachycardia from the distal great cardiac vein," Journal of the American College of Cardiology, vol. 48, no. 9, pp. 1813-1817, 2006.

[18] K. Kurzidim, H. J. Schneider, M. Kuniss et al., "Cryocatheter ablation of right ventricular outflow tract tachycardia," Journal of Cardiovascular Electrophysiology, vol. 16, no. 4, pp. 366-369, 2005.

[19] H. Aoyama, H. Nakagawa, J. V. Pitha et al., "Comparison of cryothermia and radiofrequency current in safety and efficacy of catheter ablation within the canine coronary sinus close to the left circumflex coronary artery," Journal of Cardiovascular Electrophysiology, vol. 16, no. 11, pp. 1218-1226, 2005.

[20] H. F. Tse, S. Reek, C. Timmermans et al., "Pulmonary vein isolation using transvenous catheter cryoablation for treatment of atrial fibrillation without risk of pulmonary vein stenosis," Journal of the American College of Cardiology, vol. 42, no. 4, pp. 752-758, 2003.

[21] T. Rostock, C. Weiss, R. Ventura, and S. Willems, "Pulmonary vein isolation during atrial fibrillation using a circumferential cryoablation catheter," PACE-Pacing and Clinical Electrophysiology, vol. 27, no. 7, pp. 1024-1025, 2004.

[22] Y. Van Belle, P. Janse, M. J. Rivero-Ayerza et al., "Pulmonary vein isolation using an occluding cryoballoon for circumferential ablation: feasibility, complications, and short-term outcome," European Heart Journal, vol. 28, no. 18, pp. 22312237, 2007.

[23] T. Neumann, J. Vogt, B. Schumacher et al., "Circumferential pulmonary vein isolation with the cryoballoon technique. results from a prospective 3-center study," Journal of the American College of Cardiology, vol. 52, no. 4, pp. 273-278, 2008.

[24] H. Ahmed, P. Neuzil, J. Skoda et al., "The permanency of pulmonary vein isolation using a balloon cryoablation catheter," Journal of Cardiovascular Electrophysiology, vol. 21, no. 7, pp. 731-737, 2010.

[25] K. R. J. Chun, B. Schmidt, A. Metzner et al., "The 'single big cryoballoon' technique for acute pulmonary vein isolation in patients with paroxysmal atrial fibrillation: a prospective observational single centre study," European Heart Journal, vol. 30, no. 6, pp. 699-709, 2009.

[26] G. Klein, H. Oswald, A. Gardiwal et al., "Efficacy of pulmonary vein isolation by cryoballoon ablation in patients with paroxysmal atrial fibrillation," Heart Rhythm, vol. 5, no. 6, pp. 802806, 2008.

[27] M. Mansour, G. B. Forleo, A. Pappalardo et al., "Combined use of cryoballoon and focal open-irrigation radiofrequency ablation for treatment of persistent atrial fibrillation: results from a pilot study," Heart Rhythm, vol. 7, no. 4, pp. 452-458, 2010.

[28] C. H. Siklódy, J. Minners, M. Allgeier et al., "Cryoballoon pulmonary vein isolation guided by transesophageal echocardiography: novel aspects on an emerging ablation technique," Journal of Cardiovascular Electrophysiology, vol. 20, no. 11, pp. 1197-1202, 2009.

[29] A. Fürnkranz, K. R. J. Chun, D. Nuyens et al., "Characterization of conduction recovery after pulmonary vein isolation 
using the "single big cryoballoon" technique," Heart Rhythm, vol. 7, no. 2, pp. 184-190, 2010.

[30] M. Kühne, Y. Suter, D. Altmann et al., "Cryoballoon versus radiofrequency catheter ablation of paroxysmal atrial fibrillation: biomarkers of myocardial injury, recurrence rates, and pulmonary vein reconnection patterns," Heart Rhythm, vol. 7, no. 12, pp. 1770-1776, 2010.

[31] G. B. Chierchia, C. de Asmundis, S. A. Müller-Burri et al., "Early recovery of pulmonary vein conduction after cryoballoon ablation for paroxysmal atrial fibrillation: a prospective study," Europace, vol. 11, no. 4, pp. 445-449, 2009.

[32] D. L. Packer, J. M. Irwin, J. Champagne et al., "Cryoballoon ablation of pulmonary veins for paroxysmal atrial fibrillation: first results of the North American Arctic Front stop-AF clinical trial," American College of Cardiology Annual Scientific Section, Atlanta, Ga, USA, 2010.

[33] J. N. Ruskin, J. M. Irwin, J. Champagne et al., "North American STOP-AF trial: outcomes assessments," Heart Rhythm Society Annual Scientific Section, Denver, Colo, USA, May 2010.

[34] H. Malmborg, S. Lönnerholm, and C. Blomström-Lundqvist, "Acute and clinical effects of cryoballoon pulmonary vein isolation in patients with symptomatic paroxysmal and persistent atrial fibrillation," Europace, vol. 10, no. 11, pp. 1277-1280, 2008.

[35] P. Kojodjojo, M. D. O’Neill, P. B. Lim et al., "Pulmonary venous isolation by antral ablation with a large cryoballoon for treatment of paroxysmal and persistent atrial fibrillation: medium-term outcomes and non-randomised comparison with pulmonary venous isolation by radiofrequency ablation," Heart, vol. 96, no. 17, pp. 1379-1384, 2010.

[36] Y. Van Belle, P. Janse, D. Theuns, T. Szili-Torok, and L. Jordaens, "One year follow-up after cryoballoon isolation of the pulmonary veins in patients with paroxysmal atrial fibrillation," Europace, vol. 10, no. 11, pp. 1271-1276, 2008.

[37] H. Ahmed, P. Neuzil, A. d'Avila et al., "The esophageal effects of cryoenergy during cryoablation for atrial fibrillation," Heart Rhythm, vol. 6, no. 7, pp. 962-969, 2009.

[38] T. Neumann, M. Kuniss, G. Conradi et al., "MEDAFI-Trial (Micro-embolization during ablation of atrial fibrillation): comparison of pulmonary vein isolation using cryoballoon technique vs. radiofrequency energy," Europace, vol. 13, no. 1, pp. 37-44, 2011.

[39] A. Sorgente, G. B. Chierchia, C. De Asmundis et al., "Pulmonary vein ostium shape and orientation as possible predictors of occlusion in patients with drug-refractory paroxysmal atrial fibrillation undergoing cryoballoon ablation," Europace, vol. 13, no. 2, pp. 205-212, 2011.

[40] M. Tang, C. Kriatselis, S. Nedios et al., "A novel cryoballoon technique for mapping and isolating pulmonary veins: a feasibility and efficacy study," Journal of Cardiovascular Electrophysiology, vol. 21, no. 6, pp. 626-631, 2010.

[41] G. B. Chierchia, L. Capulzini, S. Droogmans et al., "Pericardial effusion in atrial fibrillation ablation: a comparison between cryoballoon and radiofrequency pulmonary vein isolation," Europace, vol. 12, no. 3, pp. 337-341, 2010.

[42] M. Linhart, B. Bellmann, E. Mittmann-Braun et al., "Comparison of cryoballoon and radiofrequency ablation of pulmonary veins in 40 patients with paroxysmal atrial fibrillation: a case-control study," Journal of Cardiovascular Electrophysiology, vol. 20, no. 12, pp. 1343-1348, 2009.

[43] A. Luik, M. Merkel, D. Hoeren, T. Riexinger, M. Kieser, and C. Schmitt, "Rationale and design of the FreezeAF trial: a randomized controlled noninferiority trial comparing isolation of the pulmonary veins with the cryoballoon catheter versus open irrigated radiofrequency ablation in patients with paroxysmal atrial fibrillation," American Heart Journal, vol. 159, no. 4, pp. 555-560.e1, 2010.

[44] D. J. Wilber, C. Pappone, P. Neuzil et al., "Comparison of antiarrhythmic drug therapy and radiofrequency catheter ablation in patients with paroxysmal atrial fibrillation: a randomized controlled trial," JAMA-Journal of the American Medical Association, vol. 303, no. 4, pp. 333-340, 2010. 


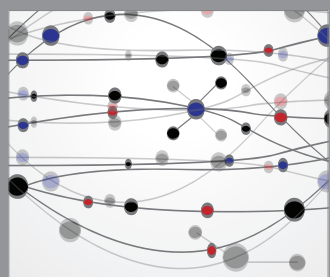

The Scientific World Journal
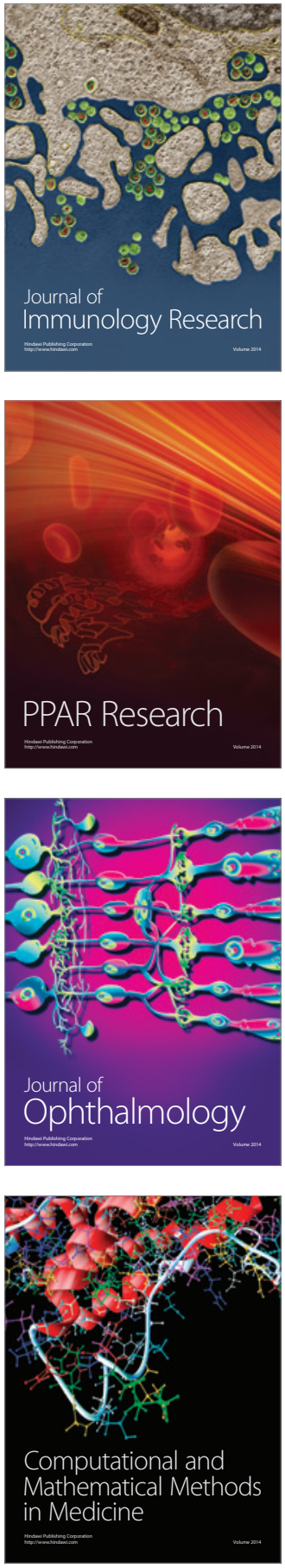

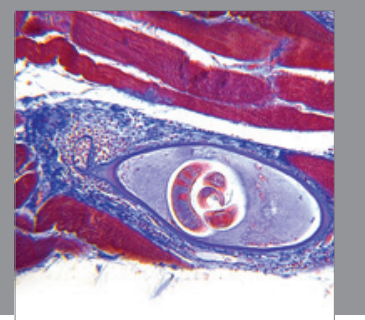

Gastroenterology

Research and Practice
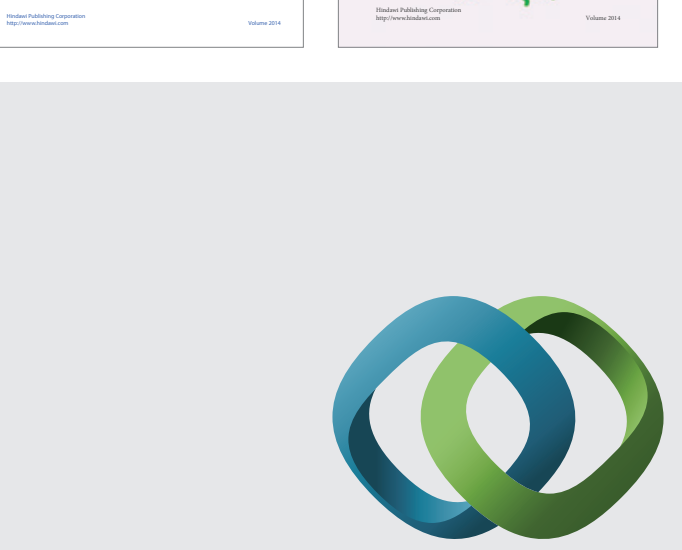

\section{Hindawi}

Submit your manuscripts at

http://www.hindawi.com
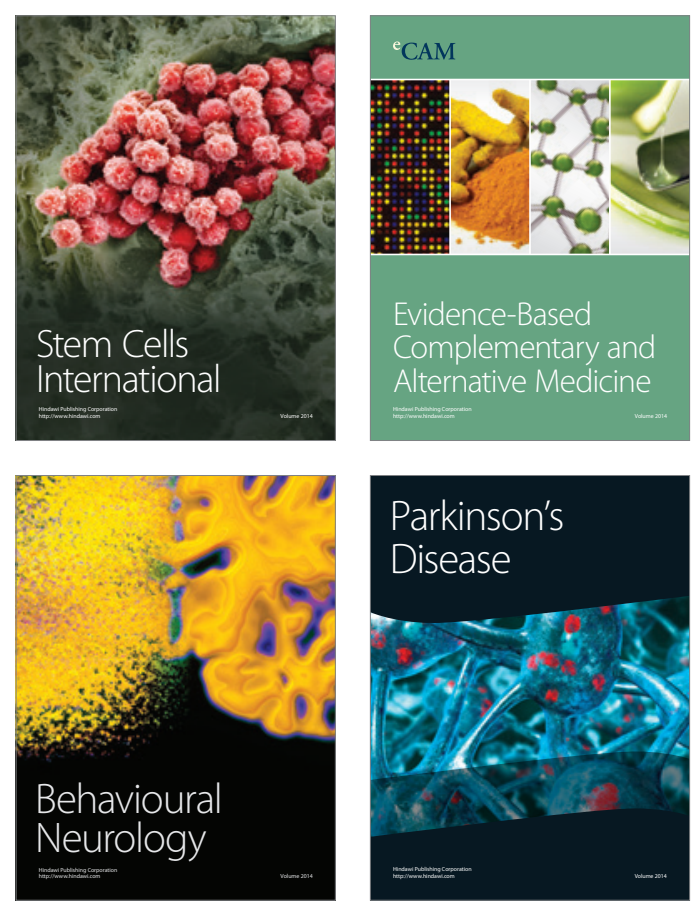

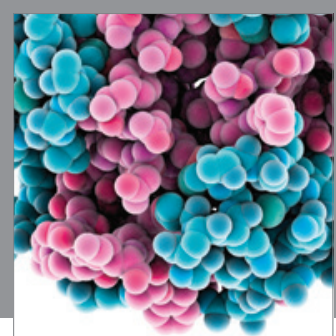

Journal of
Diabetes Research

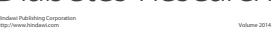

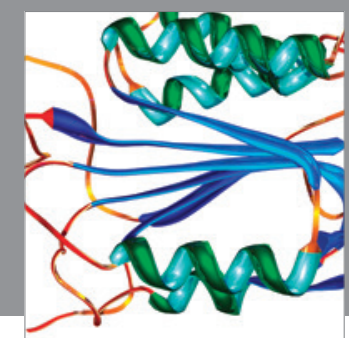

Disease Markers
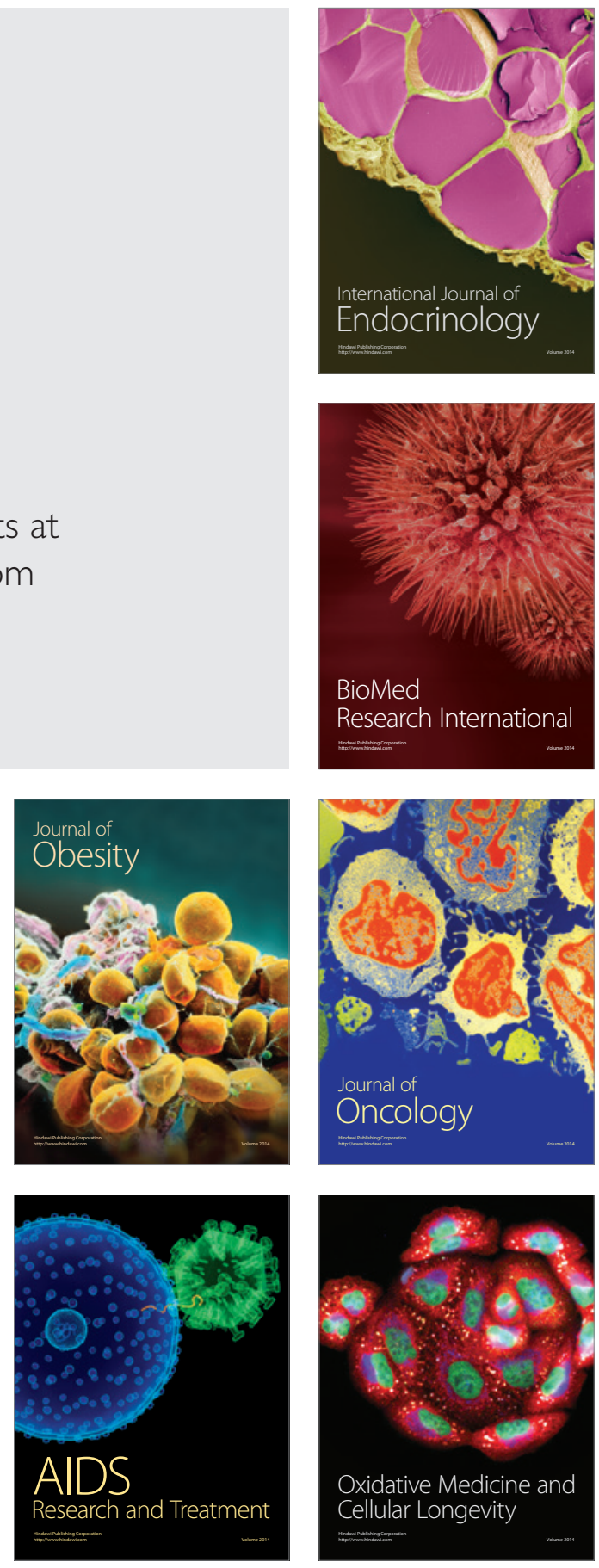